\title{
Designing a Downsized Quadrature Directional Coupler
}

\author{
Denis A. Letavin
}

\begin{abstract}
To miniaturize directional couplers, the replacement of their quarter-wave sections with electrodynamic structures with equivalent characteristics at smaller physical dimensions is used. It is shown that the structures used have filtering properties and act as low-pass filters. It is set at what cutoff frequency the low-pass filters must be calculated to obtain a phase shift of 90 degrees.
\end{abstract}

Keywords - Directional coupler, dielectric constant, microstrip line, substrate.

\section{INTRODUCTION}

$\mathrm{D}$ IRECTIONAL couplers are the basic elements of devices such as beamforming circuits, phase shifters, power dividing and summing devices, mixers, and more. However, in the classical design, the couplers are implemented in quarter-wavelength sections, the dimensions of which are related to the parameters of the substrate and the wavelength. The lower the frequency, the higher the value of the wavelength and, as a consequence, the coupler assembled on such segments will occupy a significant area and it is necessary to reduce them. The same is observed with a decrease in the dielectric constant of the material of the used substrate. To reduce the overall dimensions, it is necessary to apply various design solutions. In addition, in the process of miniaturization, it is necessary to maintain the characteristics of the compact device at the level of the classical design. Therefore, when miniaturizing a directional coupler, it is important to strike a tradeoff between size reduction and performance degradation. The IEEE scientometric database contains a large number of works related to the miniaturization of devices [1] - [9]. In [1], miniaturization is achieved by adding idle stubs, in [2], the branching of $\pi$-form transmission lines made it possible to reduce the size of a power divider operating at two different frequencies, in [3], [5], [9], replacing quarter-wave sections with artificial lines from high-resistance lines and stubs made it possible to obtain a compact coupler, in [4], [6], defects in the screen are used, in [7] low-pass filters are used to miniaturize the

Paper received April 19, 2021; accepted May 31, 2021. Date of publication July 31,2021 . The associate editor coordinating the review of this manuscript and approving it for publication was Prof. Branko Kolundžija.

This paper is a revised and expanded version of the paper presented at the 28th Telecommunications Forum TELFOR 2020 [10].

Letavin Denis A. The engineer of department of high-frequency devices for radio communication and television of Institute of radio electronics and information technology of the Ural federal university, finished this university in 2020 on a specialty "Radioengineering". Scientific interests - applying electrodynamics (e-mail: d.a.letavin@urfu.ru). coupler, in [8] to miniaturize the separator, bends of lines into the internal space of the device are used. Each approach produces its own results in miniaturizing directional couplers. Taps with two and three plug-in stubs are widely used, since with a larger number of stubs, their wave impedances become so high that it is difficult to implement in practice. Therefore, little attention is paid to the issues of miniaturization of taps with the number of connected stubs more than three. In our work, we have proposed a design of a four-loop directional coupler, in which high-resistance stubs are implemented on lumped elements, which increases the degree of miniaturization of the device.

\section{COUPLERS DESIGN}

The purpose of this paper is to describe the development process of a compact directional coupler with four pluggable stubs. Additional connection of stubs to the design of the coupler allows you to expand its operating frequency band. However, in addition to this, the addition of stubs leads to an increase in the size of the device and the need to miniaturize the coupler increases. Fig. 1 shows a diagram of a coupler with four connected stubs, where: Z1 is equal to $48.6 \Omega ; \mathrm{Z} 2$ is equal to $50.85 \Omega ; \mathrm{Z} 3$ equals $131 \Omega$ ohms; Z4 equals $122.4 \Omega$ ) and the indicated characteristic impedances.

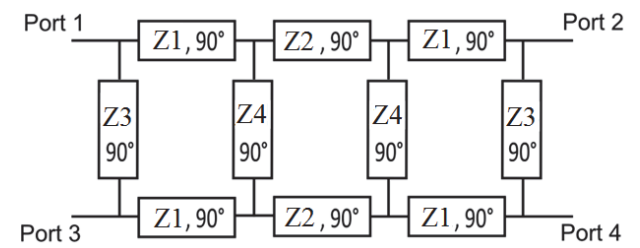

Fig. 1. Diagram of a four-line directional coupler.

FR4 (parameters) acts as a substrate material, and $2 \mathrm{GHz}$ acts as an operating frequency. Using the formulas, the dimensions of the quarter-wave sections of the directional coupler were obtained. Without taking into account the supply lines, the dimensions of the coupler are $1798 \mathrm{~mm}^{2}$ (Fig. 2). Using the Cadence AWR DE software, the frequency characteristics of a classic coupler were calculated. The calculated graphs for a standard coupler are shown in Figs. 3 and 4.

According to the obtained frequency dependences, such parameters of the coupler were determined as the operating frequency band (estimated by the isolation level of $15 \mathrm{~dB}$ ) equals to $1450 \mathrm{MHz}$. In the same band, a phase difference of the output signals of $\sim 90^{\circ}$ is provided. An imbalance between gains of $\pm 0.5 \mathrm{~dB}$ remains in the $740 \mathrm{MHz}$ frequency band. 


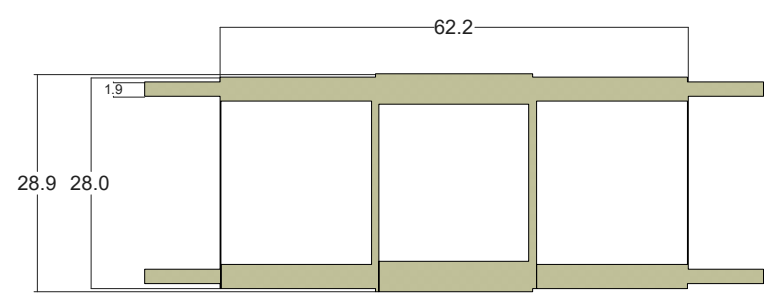

Fig. 2. Layout of a traditional directional coupler.

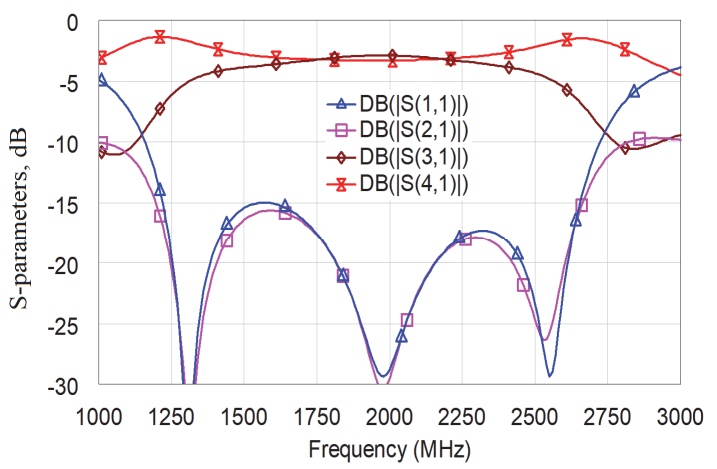

Fig. 3. Graph of S-parameters from the frequency of the coupler.

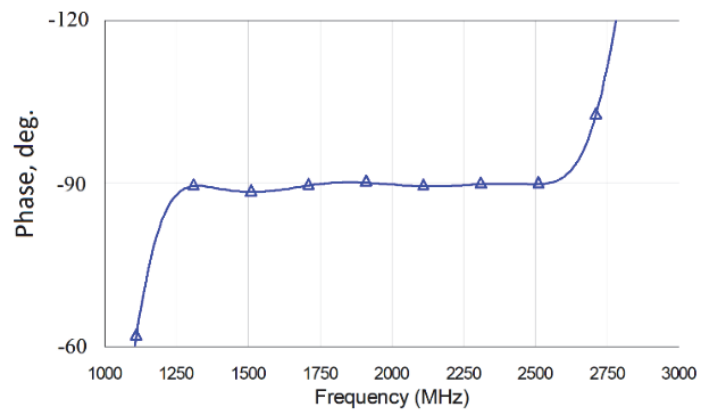

Fig. 4. Graph of the phase difference of the output signals of the coupler.

Fig. 2 shows that the area inside the coupler enclosed between the quarter-wave sections is not used in any way. This area will be effectively used when miniaturizing the device. Using the values of the characteristic impedances of quarter-wave sections, it is possible to calculate structures that have equivalent characteristics at smaller sizes. The phase velocity and characteristic impedance of the transmission line are related by the following relationships:

$$
\begin{aligned}
\rho & =\sqrt{\frac{L}{C}} \\
v_{\phi} & =\frac{1}{\sqrt{L C}}
\end{aligned}
$$

The wavelength and phase velocity are related by the relationship:

$$
\lambda=\frac{v_{\phi}}{f}
$$

From (2), it is obvious that with an increase in capacitance in an equivalent section of a long line, it will lead to a decrease in the phase velocity of an electromagnetic wave, due to which the wavelength will also decrease, according to (3). This fact makes it possible to reduce the dimensions of microwave devices, in our case, stubs quadrature couplers. Fig. 5 illustrates the layout of the structure, and Fig. 6 shows its amplitude-frequency characteristics.

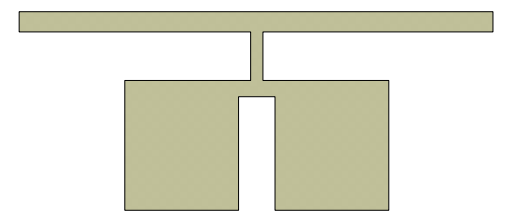

Fig. 5. Structure layout.

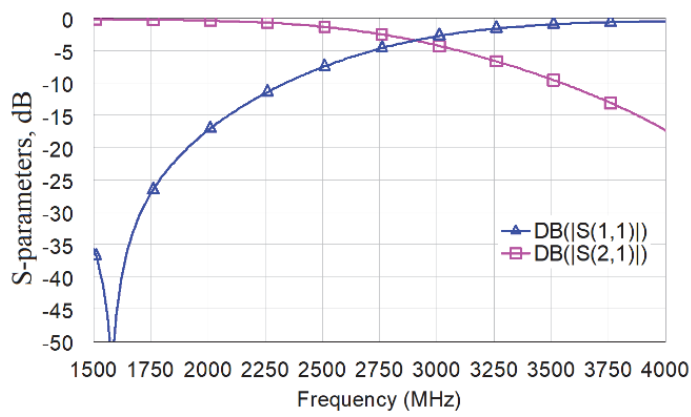

Fig. 6. Frequency response of the structure.

However, it is necessary to determine at what cutoff frequency the low-pass filter must be calculated in order for it to provide a phase shift of 90 degrees. Let's construct the frequency response and phase response for the simplest low-pass filter (LPF) and high-frequency filter (HPF), shown in Fig. 7.
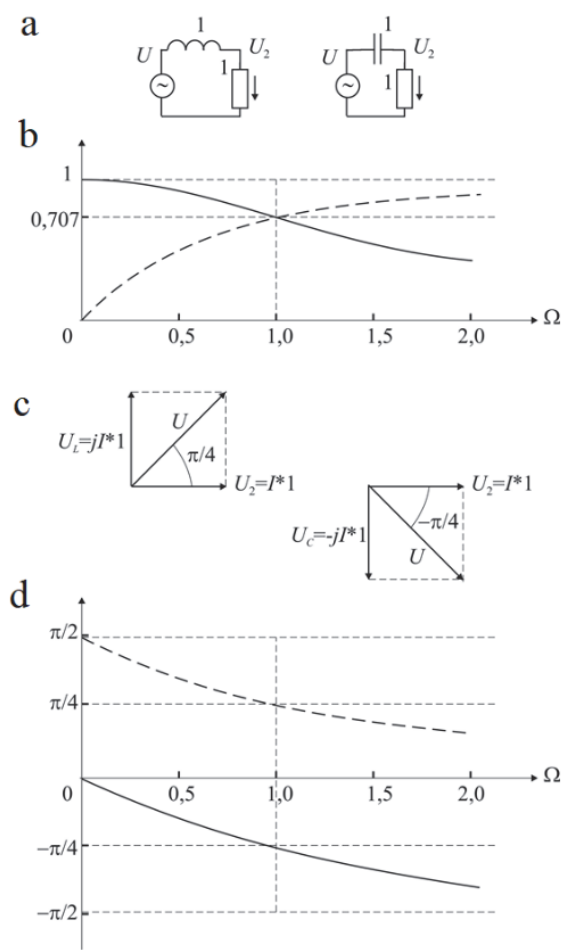

Fig. 7. Frequency response and phase response of the simplest LPF and HPF.

In Fig. 7, the frequency characteristics of the low-pass filter are depicted by solid lines, the frequency characteristics of the high-pass filter are dashed. In particular, Fig. $7 \mathrm{~b}$ shows the amplitude-frequency characteristic of the LPF and HPF at the same cutoff frequencies. Fig. 7c shows the vector diagrams of voltages and currents constructed for the frequency $\Omega=1$. 
Let's carry out similar constructions for the normalized low-pass filter of the third order (Fig. 8).
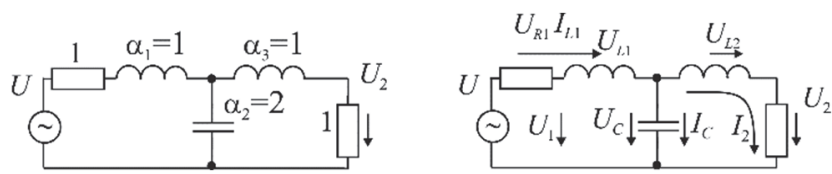

Fig. 8. LPF (on the left with the designations of the elements, on the right with the designations of currents and voltages).

When constructing, the following assumptions were made:

- the voltage across the inductance is determined by the ratio $\dot{U}_{L}=\dot{I}_{L} x_{L}=\dot{I}_{L} j \Omega \alpha_{L}=\dot{I}_{L} j \Omega=\dot{I}_{L} e^{(j \pi / 2)} \Omega$ (since $\alpha_{1}=\alpha_{3}=$

1 ), that is, it advances the current by an angle of $90^{\circ}$;

- the capacitor current is determined by the ratio

$\dot{I}_{C}=\dot{U}_{C} /\left(1 / j \Omega \alpha_{2}\right)=j \Omega \alpha_{2} \dot{U}_{C}=j 2 \Omega \dot{U}_{C}=2 \Omega \dot{U}_{C} e^{(j \pi / 2)}$

(since $\alpha_{C}=2$ ), that is, it advances the voltage by an angle of $90^{\circ}$.

Construction of a vector diagram at a frequency $\Omega=1$. "Each step" in the diagram is marked in the figure with a number in a circle:

1. Let's take the value of the current $I_{2}=1 . U_{2}=I_{2} \cdot 1=1$ or $U_{2}=I_{2}=1 . e^{j 0^{\circ}}=\cos 0^{\circ}+j \sin 0^{\circ}=1$. Accordingly, we represent this unit vector coinciding in direction with the $\mathrm{x}$ axis.

2. $\dot{U}_{L 2}=\dot{I}_{2} j \Omega \alpha_{3}=j 1=e^{(j \pi / 2)}=\cos (\pi / 2)+j \sin (\pi / 2)=j 1$ This

is a vector leading the vector $U_{2}$ by an angle $\pi / 2$.

3. $U_{C}$ is the sum of vectors $U_{2}$ and $U_{L 2}$.

$\dot{U}_{C}=\dot{U}_{2}+\dot{U}_{L}=1+j 1=\sqrt{2} e^{j \phi_{1}} \cdot \phi_{1}=\operatorname{arctg} 1=\pi / 4$

$\dot{U}_{C}=\sqrt{2} e^{j \pi / 4}$. Construction as a vector sum of $U_{2}$ and $U_{L 2}$.

$$
\text { 4. } \dot{I}_{C}=\dot{U}_{C} /\left(1 / j \Omega \alpha_{2}\right)=\sqrt{2} e^{j \pi / 4} j 2=2 \sqrt{2} e^{(j 3 \pi / 4)}=-2+j 2 \text {. }
$$

This vector can be plotted by plotting " +2 " along the vertical axis, and "-2" along the horizontal.

5. $I_{L 1}$ is the sum of vectors $I_{2}$ and $I_{C}$.

$\dot{I}_{L 1}=1-2+j 2=-1+j 2=\sqrt{5} e^{j \phi_{2}} \cdot \varphi_{2}=\operatorname{arctg}(-2)$

It can also be plotted by plotting " $+2 "$ along the vertical axis, and "-1" along the horizontal axis (or as the sum of vectors).

6 . The vector is plotted by plotting "-2" on the $\mathrm{x}$-axis and "- 1 " on the y axis.

$\dot{U}_{L 1}=\dot{I}_{L 1} j \Omega \alpha_{1}=\dot{I}_{L 1}\left(e^{j \pi / 2}\right)=(-1+j 2)(j 1)=-2-j 1=\sqrt{5} e^{\left(j \phi_{3}\right)}$

$\varphi_{3}=\operatorname{arctg}(1 / 2)$

7. $U_{1}$ is the sum of vectors $U_{L 1}$ and $U_{C}$.

$\dot{U}_{1}=-2-j 1+j 1+1=-1$. Deferred in the figure under the number "7".

8. The vector of the electromotive force source $U$ is found as the sum of the vectors $U_{R 1}$ and $U_{1}$. Let us take into account that the vector $U_{R 1}$ coincides in magnitude and direction with the vector $I_{L 1}$, since $U_{R 1}=I_{L 1} \cdot 1$ (1 is the internal resistance of the source, see Fig. 3).

$\dot{U}=U_{R 1}+U_{1}=j 2-1-1=j 2-2$, that is, it coincides in magnitude and direction with the $\mathrm{I}_{\mathrm{C}}$ vector.

Thus, the vector $U$ leads the vector $U_{2}$ by an angle of
$3 \pi / 4=135^{\circ}$ or that the same output voltage $U_{2}$ lags behind the input action $\mathrm{U}$ by an angle of $135^{\circ}$. If necessary, all angles $\varphi 1, \varphi 2, \varphi 3$ can be calculated taking into account the quadrant in which it (the angle) falls and build a diagram using them (Fig. 9).

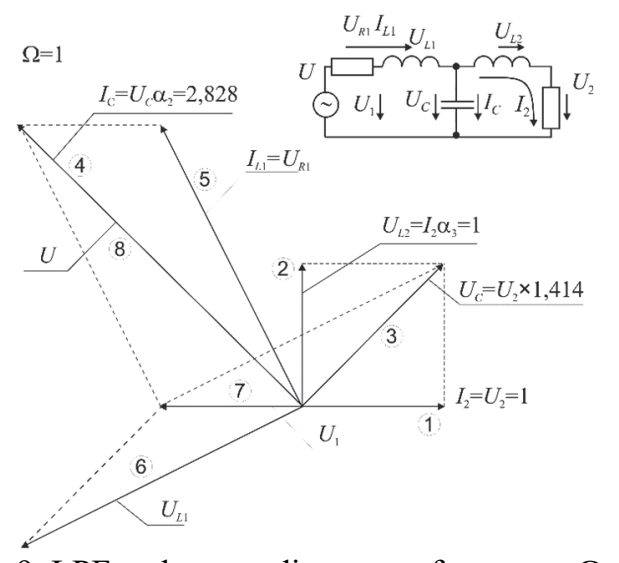

Fig. 9. LPF and vector diagram at frequency $\Omega=1$.

After completing steps " 1 " to " 8 " for $\Omega=1 / \sqrt{ } 2$, we obtain a phase shift of $\pi / 2=90^{\circ}$. Phase characteristics for LPF and HPF are shown in Fig. 10, from which it follows that the frequencies at which the phase shifts of the filters are equal to $\pm 90^{\circ}$ differ by exactly two times.

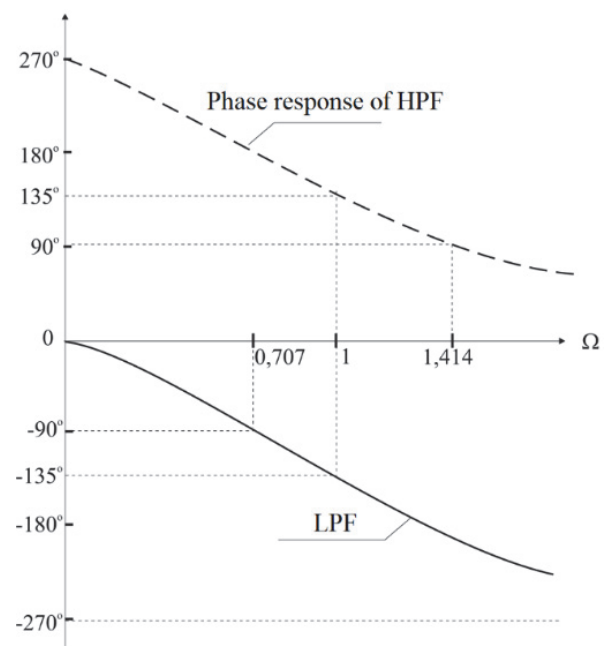

Fig. 10. Phase response for LPF and HPF.

For example, if it is necessary to obtain phase shifts $\pm 90^{\circ}$ at a frequency of $1000 \mathrm{MHz}$, it is necessary to denormalize (calculate) the LPF relative to the frequency of $1414 \mathrm{MHz}$, and the HPF relative to the frequency of $707 \mathrm{MHz}$, as shown in Fig. 11.

The next step is to use well-known expressions to perform the transition from lumped elements to distributed ones. However, it is more advantageous to bend highresistance segments while maintaining their length than to replace them with electrodynamic structures, which will be impossible to implement using standard equipment. After that, we get the layout of a compact four-loop coupler (Fig. 12) and the frequency characteristics obtained using the Cadence AWR (Figs. 13, 14).

From the obtained frequency dependences, it can be seen that the compact coupler operates in a frequency band (estimated by the isolation level of $15 \mathrm{~dB}$ ) equal to 
$870 \mathrm{MHz}$. In the same band, a phase difference of the output signals of $\sim 90^{\circ}$ is provided. An imbalance between gains of $+0.5 \mathrm{~dB}$ remains in the $700 \mathrm{MHz}$ frequency band. In this case, the area of the coupler turned out to be $69.9 \%$ less and is $541 \mathrm{~mm}^{2}$. The deterioration of characteristics is due to the fact that the characteristic impedance of the structures changes when the operating frequency changes. Comparison of classic and compact coupler is shown in table 1 .

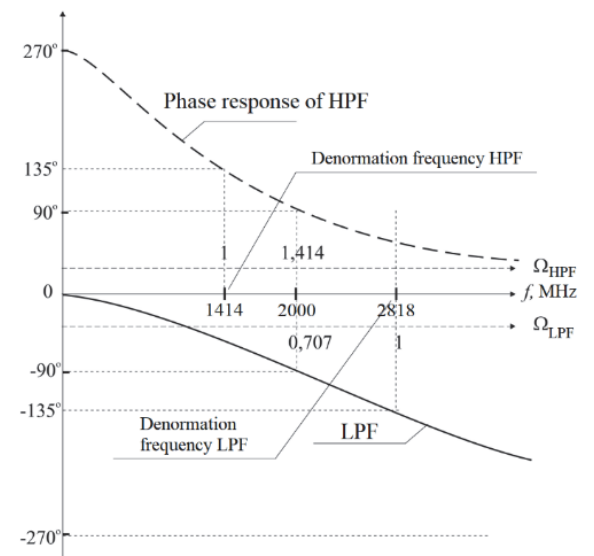

Fig. 11. Matched phase response LPF and HPF.

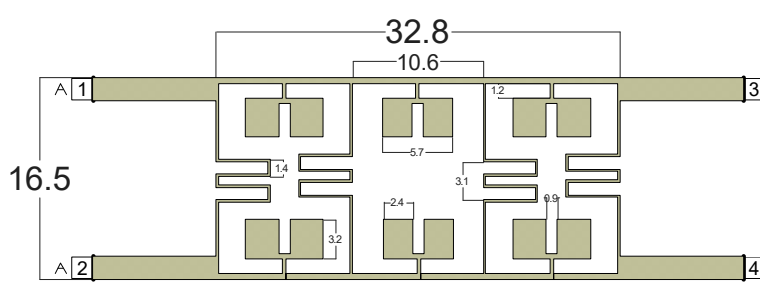

Fig. 12. Layout of a compact directional coupler.

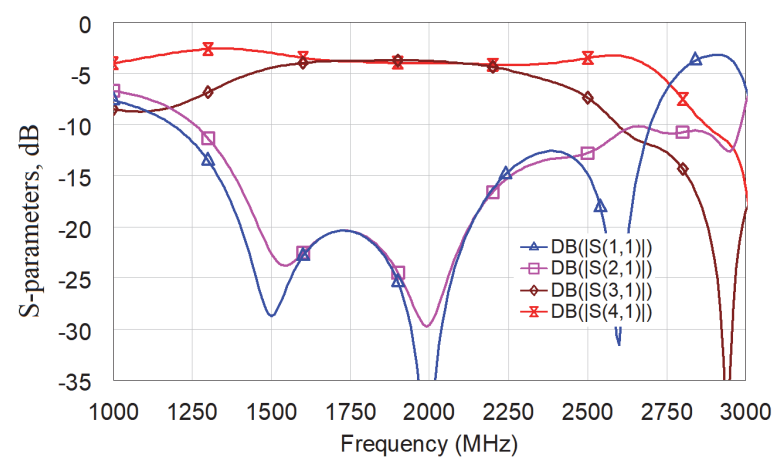

Fig. 13. Graph of S-parameters from the frequency of the coupler.

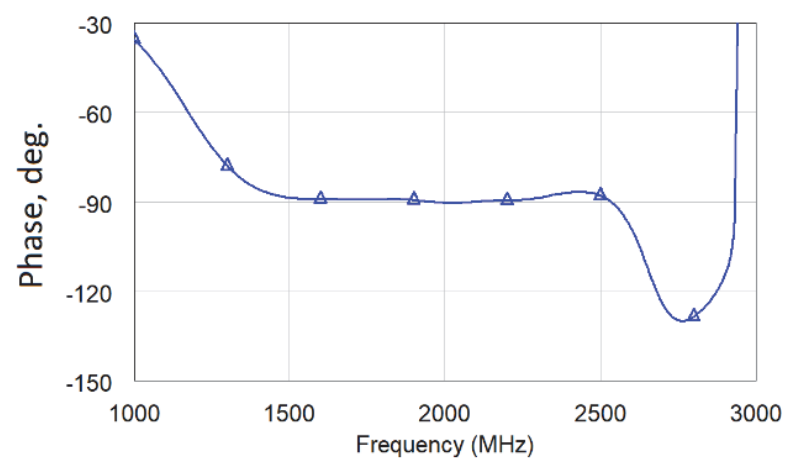

Fig. 14. Graph of the phase difference of the output signals of the coupler.
TABLE 1: COMPARISON OF CLASSIC VERSUS MINIATURE DIRECTIONAL COUPLER.

\begin{tabular}{c|c|c}
\hline Parameters & Standard & Compact \\
\hline bandwidth, $\mathrm{MHz}$ & 870 & 856 \\
Relative bandwidth, \% & 43.5 & 42.8 \\
area, $\mathrm{mm}^{2}$ & 1798 & 541 \\
Relative area, \% & 100 & 30.1 \\
Central frequency, $\mathrm{MHz}^{2}$ & 2000 & 2000 \\
The phase outputs, $^{\circ}$ & 90 & 89.4 \\
\hline
\end{tabular}

\section{CONCLUSION}

In this work, a study was carried out of a four-loop directional coupler with reduced overall dimensions using electrodynamic structures with equivalent characteristics with quarter-wave sections. The stages of calculating a compact four-loop coupler are described. Replacing highresistance sections with lumped elements further increases the degree of miniaturization of the coupler. The advantage of the obtained design of the coupler consists in a significant reduction in the dimensions of the device. The area of the proposed coupler layout corresponds to $30 \%$ of the area of the classic design.

\section{REFERENCES}

[1] Liangfan Zhu, "Compact Branch-line Coupler using Radial stubs," 2019 Cross Strait Quad-Regional Radio Science and Wireless Technology Conference (CSQRWC). DOI: 10.1109/CSQRWC.2019.8799273.

[2] Chao Gai, Yong-Chang Jiao and Yu-Long Zhao, "Compact DualBand Branch-Line Coupler With Dual Transmission Lines," IEEE Microwave and Wireless Components Letters, vol. 26, no.5, pp. 325 $327,2016$.

[3] Vamsi Krishna Velidi, Sarika Shrivastava and Subrata Sanyal, "A compact-size microstrip rat-race coupler with high performance,' IEEE Technology Students' Symposium. DOI: 10.1109/TECHSYM.2011.5783801

[4] Y. J. Sung, C. S. Abn, and Y. S. Kim, "Size reduction and harmonic suppression of rat-race hybrid coupler using defected ground structure," IEEE Microw. Wireless Compon. Lett., vol. 14, no. 1, pp. 7-9, Jan. 2004.

[5] J. T. Kuo, J. S. Wu, and Y. C. Chiou, "Miniaturized rat-race coupler with suppression of spurious passband," IEEE Microw. Wireless Compon. Lett., vol. 17, no. 1, pp. 46-48, Jan. 2007.

[6] Huaming Wang, Xueguang Liu, Wenfeng Cai and Hongfang Cao, "Design and realization of a new compact branch-line coupler using defected ground structure," 2008 9th International Conference on Solid-State and Integrated-Circuit Technology, 2008, doi: 10.1109/ICSICT.2008.4734818.

[7] D. A. Letavin, Y. E. Mitelman, and V. A. Chechetkin, "A Novel Simple Miniaturization Technique for Double Rat-race coupler," 2019 Antennas Design and Measurement International Conference, ADMInC, 2019, doi: 10.1109/ADMInC47948.2019.8969084.

[8] Ashmi Chakraborty Das, Lakhindar Murmu and Santanu Dwari, "A Compact Branch-Line Coupler using Folded Microstrip Lines," 2013 International Conference on Microwave and Photonics (ICMAP), 2013.

[9] K. W. Eccleston and S. H. M. Ong, "Compact planar microstripline branch-line and rat-race couplers," IEEE Trans. Microw. Theory Techn., vol. 51, no. 10, pp. 2119-2125, Oct. 2003.

[10] D. A. Letavin, "Bandwidth Miniature Quadrature Branch line Coupler Design," 2020 28th Telecommunications Forum (TELFOR), 2020, pp. 1-3, doi: 10.1109/TELFOR51502.2020.9306606. 